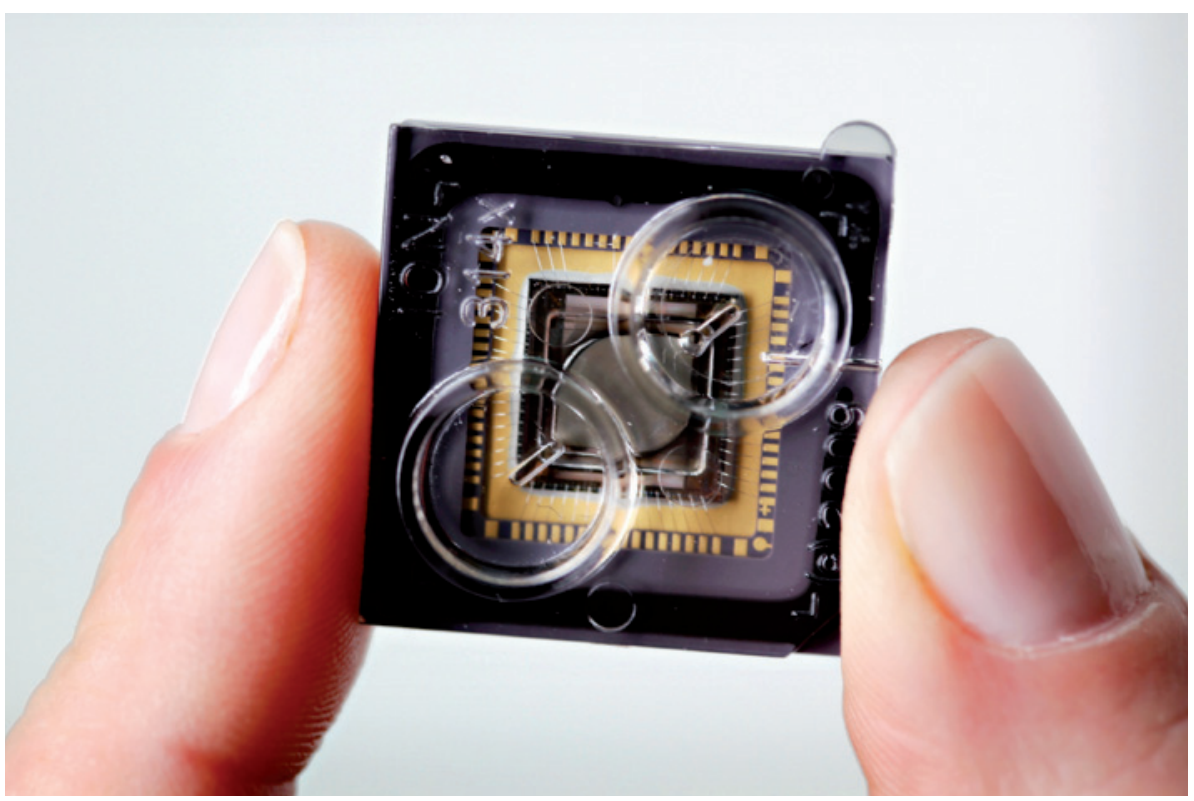

Each Ion Torrent chip sports 1.2 million DNA-testing wells.

\title{
GENOMICS
}

\section{Chip chips away at the cost of a genome}

\section{Ion-sensing method offers cheap sequencing in record time.}

\section{BY GWYNETH DICKEY ZAKAIB}

$\mathrm{T}$ he latest contender in the race for the prized ' $\$ 1,000$ genome' has proved its mettle in a singularly appropriate way: by sequencing the genome of computer pioneer Gordon Moore.

Like the computer chips made by Intel, the company that Moore co-founded, the Ion Personal Genome Machine (PGM) exploits semiconductor technology, with its ability to deliver ever-increasing speed and lower costs - a trend predicted by 'Moore's law' some 50 years ago. When Ion Torrent of Guilford, Connecticut, part of Life Technologies in Carlsbad, California, introduced the device late last year ${ }^{1}$, some scientists wondered whether it could live up to its promise to put a sequencer within the reach of any reasonably funded lab. Their doubts are likely to wane in the wake of the company's latest demonstration, published this week in Nature (see page 348).

In addition to producing a rough draft of Moore's genome, Ion Torrent has shown that its US $\$ 49,500$ device can read a bacterial genome in as little as two hours. "It's a quantum leap in terms of the time it takes to do an experiment," says Stephan Schuster, a molecular biologist at Pennsylvania State University in University Park, who has been testing the technology

for nearly a year. He has already published a paper that uses ion-sequencing data to investigate a cancer that is spreading rapidly among Tasmanian devils ${ }^{2}$. The PGM was also the first platform to unravel the genome of the Escherichia coli strain that began wreaking havoc in Germany in $\mathrm{May}^{3}$, delivering a sequence in just three days.

What makes the technology so quick and inexpensive is the novel way it detects the identity of the nucleotide bases in DNA. The Human Genome Project, which unveiled its landmark results a decade ago, relied on the laborious Sanger sequencing method. This involves building complementary DNA strands to match the original sample, until nucleotides labelled with a fluorescent dye are added to halt the process. The copied fragments are then sorted by size to determine the sequence of the original strand.

More recently, faster 'next generation' techniques were developed to read a DNA sequence by tracking the construction of a complementary strand as it actually happens. Most

\section{$\rightarrow$ NATURE.COM}

For a special on the human genome at ten, see: go.nature.com/ugle41 methods use fluorescent labelling to identify individual nucleotides as they are added. But these reagents are expensive each sequencing run can cost thousands of dollars, and may still take more than a week to complete.

Ion Torrent's device instead uses cheaper, natural nucleotides, and senses the hydrogen ions (protons) that are released as each nucleotide is incorporated onto the complementary DNA. "We made an array that literally sees chemistry," says molecular biologist Jonathan Rothberg, chief executive of Ion Torrent.

Microscopic beads carrying fragments of DNA are first loaded into 1.2 million 3.5-micrometre-wide wells covering a small chip that cost $\$ 99$. The chip is then flooded with washes of different nucleotides bearing the four bases that make up DNA, one after another. The wells are cleaned between each wash. If a nucleotide is complementary to the next unpaired base on the bead, it binds and gives off a hydrogen ion, changing the $\mathrm{pH}$ inside the well. This produces an electrical signal, indicating that the base in that particular wash is the next letter of the sequence. Each step takes less than five seconds, enabling a single chip to read about 25 million bases in a single two-hour run, and for just a few hundred dollars.

The technology's utility will ultimately depend not only on its cost, but also its accuracy, says Stephen Chanock, head of the Laboratory of Translational Genomics at the National Cancer Institute in Bethesda, Maryland. And, on that front, the PGM is still no match for the biggest, most expensive machines. Costing hundreds of thousands of dollars, they can read hundreds of billions of base pairs in a single run, and they are currently a more appropriate choice for tackling whole human genomes with high fidelity. Ionchip sequencing is better suited to achieving fast results in smaller-scale projects, such as sequencing bacterial genomes or characterizing diseases by reading certain gene regions across many patients.

But Rothberg emphasizes that as transistors are packed more densely onto a single chip, $\begin{array}{ll}\text { "It's a quantum } & \text { the technology will } \\ \text { leap in terms } & \text { become much more }\end{array}$ of the time it takes to do an experiment."

Moore's genome required 1,000 ion chips - totalling 1 billion sensors working in parallel. But the company is already testing an 11-million-well chip that could shrink that requirement tenfold and cut costs even further.

By switching to a manufacturing process able to create smaller features on a chip, Rothberg says, "we're very comfortable that we'll get way below a $\$ 1,000$ genome".

\footnotetext{
1. Katsnelson, A. Nature http://dx.doi.org/10.1038/ news.2010.674 (2010).

2. Miller, W. et al. Proc. Natl Acad. Sci. USA http:// dx.doi.org/10.1073/pnas.1102838108 (2011).

3. Turner, M. Nature http://dx.doi.org/10.1038/ news.2011.345 (2011).
} 